\title{
APRENDIZAJE COOPERATIVO EN OBSERVACIÓN SISTEMÁTICA MEDIANTE EL VISIONADO DE FILMS
}

Beatriz Peña-Acuña1. Universidad Católica San Antonio. España bpena@pdi.ucam.edu

M. Concepción Parra-Meroño: Universidad Católica San Antonio. España mcparra@pdi.ucam.edu

\section{RESUMEN}

En este trabajo se propone la utilización del Cine como herramienta de entrenamiento mediante la cual se puede mejorar la capacidad de la observación. Nuestro trabajo explica como el visionado de films (secuencias, extras y fragmentos de películas de cine), con su lenguaje propio, tanto verbal como no verbal, contribuye de forma innovadora al desarrollo de la capacidad de la observación sistemática. Esta innovación docente se ha aplicado en estudiantes de Magisterio en el ámbito del Espacio Europeo de Educación Superior (EEES), mediante la metodología del aprendizaje cooperativo y la tutoría en grupo. Mediante el visionado de diferentes films se introduce al estudiante en el lenguaje cinematográfico y en el lenguaje no verbal, lo que le permite desarrollar y practicar la observación de las conductas humanas, ya sea a través el trabajo individual como a través del trabajo cooperativo y apoyado con la práctica de tutorías en grupo. Todo ello a su vez, induce a reflexionar sobre diferentes contenidos del proceso de enseñanza-aprendizaje. Así, una vez adquiridas las destrezas sobre el lenguaje cinematográfico los alumnos son capaces de aplicarlas en otros productos audiovisuales sobre educación, ya sean programas específicos, debates o documentales sobre educación, tanto en radio como en televisión.

PALABRAS CLAVE: Comunicación no verbal - Proceso de enseñanza-aprendizaje Aprendizaje cooperativo - Competencias - Innovación educativa

\footnotetext{
${ }^{1}$ Autor correspondiente

Beatriz Peña-Acuña: Profesora Doctora en la Facultad de Ciencias de la Educación. Universidad Católica San Antonio. Murcia, España.
}

Correo: bpena@pdi.ucam.edu 


\title{
SYSTEMATIC OBSERVATION ON COOPERATIVE LEARNING THROUGH VIEWING FILMS
}

\begin{abstract}
This paper proposes the use of film as a training tool by which to improve the capacity of observation. Our work explains how the viewing of films (sequences, extras and excerpts from films), with its own language, both verbal and nonverbal, contributes to the development of innovative capacity for systematic observation. This innovation has been applied in teaching student teachers in the field of European Higher Education Area (EHEA), using the methodology of cooperative learning and group tutoring. By viewing different films the teacher introduces students to the cinematic language and the nonverbal language, allowing them to develop and practice the observation of human behavior, either through individual work or through the supported cooperative work and the practice of mentoring groups. This in turn makes us think about different contents of the teaching-learning process. Thus, once acquired skills on the language of film students are able to apply them in other audio-visual education, whether specific programs, debates and documentaries on education, both in radio and television.
\end{abstract}

KEY WORDS: Nonverbal communication - teaching-learning process -cooperative learning - competencies - educational innovation

\section{INTRODUCCIÓN}

La Ciencia de la Comunicación interactúa cada vez más con otras disciplinas, como en el caso de los estudios de Empresa, en los que ya se dedica una atención especial a la comunicación interna, o en el de las Ciencias de la Educación, donde actúa como medio de transmisión de valores. Así lo han venido sosteniendo en la práctica numerosos investigadores como (De la Torre, 1996); (Almacellas, 2004) y así mismo lo hemos sostenido de forma teórica, primero en una ponencia (Peña, 2004) y posteriormente apoyado por el aparato científico de una investigación de tesis doctoral (Peña, 2010). Autores como de Pablos (Pablos, 1986) también han dado un sustento científico a tal enunciado. 
A partir del conocimiento de las posibilidades que ofrece el lenguaje cinematográfico, se nos brindó la posibilidad de su utilización como una herramienta idónea dadas sus características, para contribuir al desarrollo de la observación del alumno. Por ello, decidimos utilizar un enfoque multidisciplinar en el aula, usando la Ciencia de la Comunicación en el ámbito de la enseñanza aprendizaje de la Ciencia de la Educación. Nuestro trabajo explica el uso del cine y la comunicación no verbal de forma innovadora en la experiencia educativa, como instrumento para formar y desarrollar la capacidad de observación, en alumnos de Magisterio en el marco del Espacio Europeo de Educación Superior (EEES), con todas las destrezas que esta práctica desarrolla.

A partir de la definición psicológica de observación como "el proceso de aprendizaje de nuevas respuestas a través de la observación conductual de otro" (AAVV, 2010). Nuestro propósito es que los alumnos alcancen las competencias planificadas en la asignatura de observación sistemática, según el marco de Bolonia, mediante la aplicación de nuevos métodos de enseñanza aprendizaje, en los que el estudiante se configura como el centro de su propio proceso de aprendizaje, lejos del rol que representa en las tradicionales lecciones magistrales tan utilizadas de forma tradicional en las universidades españolas.

En este contexto entendemos por competencia el conjunto el conjunto de conocimientos, actitudes y destrezas necesarias para desempeñar una función (Le Boterf, 2001). Así, las competencias se concretan en resultados de aprendizaje, y entendidas como lo hace el Proyecto Tuning, se pueden definir también como "conocimientos, habilidades, actitudes y responsabilidades, que describen los resultados de un programa educativo o lo que los alumnos son capaces demostrar al final del proceso educativo". Así, la Declaración de Bolonia sostiene que los planes de estudios deben ofrecer una formación tal que permita el desarrollo de los perfiles profesionales de la titulación. Esta formación permitirá a los alumnos la adquisición de conocimientos y habilidades, denominadas competencias, que pueden ser específicas, genéricas y transferibles (ANECA, 2005).

Así, mediante la práctica de la observación conseguíamos que obtuvieran las destrezas necesarias para ella. Entendíamos destreza como: "Habilidad, facilidad o arte para hacer algo bien hecho: Para los trabajos manuales, hay que tener destreza con las manos." Esta innovación -utilizar el cine como medio para desarrollar la observación en los alumnos- la encontramos idónea a la hora de alcanzar además determinados objetivos generales de la asignatura en cuestión (UCAM, 2009), como: actuar como mediador para que toda la actividad que se lleve a cabo resulte significativa y estimule el potencial de desarrollo de cada uno de los alumnos en un trabajo cooperativo del grupo. Ser capaz de diseñar y organizar trabajos disciplinares e interdisciplinares y de colaborar con el mundo exterior a la escuela. Ser un profesional capaz de analizar el contexto en el que se desarrolla su actividad y planificarla, y de dar respuesta a una sociedad cambiante. 
Esta metodología, permite a su vez alcanzar otras competencias, tales como el desarrollo de la sensibilidad por la belleza y el valor que otorga el alumno a un trabajo minucioso y bien hecho desarrollado a través del lenguaje cinematográfico. Mediante el entrenamiento en la observación de detalles, no solo se considera el propio lenguaje cinematográfico, sino también las cuantiosas tareas realizadas por los distintos departamentos de una producción cinematográfica.

En concreto se alcanzaban además las siguientes competencias específicas de la asignatura (UCAM, 2009): Capacidad de análisis y síntesis. Capacidad de gestión de la información. Comprender que la observación sistemática es un instrumento básico para poder reflexionar sobre la práctica y la realidad, así como contribuir a la innovación y a la mejora en educación infantil. Dominar la técnica de observación. Por ello, se consigue alcanzar también los objetivos específicos de la asignatura (UCAM, 2009): Conocer las diferentes estrategias de investigación y análisis de contextos. Comprender y aplicar la metodología observacional. Adquirir y usar la terminología específica de la metodología observacional. Saber cómo se recogen, analizan e interpretan datos observacionales. Valorar e interpretar trabajos de investigación observacional.

Para capacitar a los alumnos se utilizaron sesiones de dos tipologías: talleres prácticos en el aula y tutorías en grupos reducidos. En los primeros la participación se realizaba a través de los portavoces de los diferentes grupos, posibilitando en aprendizaje grupal y cooperativo. En las tutorías, se trabaja en grupos reducidos que no superan los 15 individuos, por lo que el aprendizaje es más significativo, y permite un seguimiento más personalizado y pormenorizado. Estas metodologías de aprendizaje cooperativo ya las habíamos utilizado con anterioridad en otras actividades académicas (Parra y Peña 2009), con buenos resultados, por lo que dada la experiencia previa, nos pareció muy adecuado su utilización en esta asignatura.

En cuanto a los beneficios que esperábamos que los alumnos adquirieran con este ejercicio audiovisual se encontraban: la capacidad de realizar hallazgos de forma autónoma y grupal, la adquisición de una mayor autoestima, una mayor conciencia de su capacidad social, una mejoría de la actitud proactiva y un desarrollo concreto de la creatividad a través del trabajo en grupo que tenían que inventarse ex novo. 


\section{METODOLOGÍA}

\subsection{La observación sistemática}

Según Croll (1995, p. 33) la observación sistemática puede entenderse como "un método de investigación que utiliza procedimientos de observación muy estructurados aplicados por observadores formados en la materia con objeto de recoger datos sobre modelos de comportamiento e interacción en clase". Siendo las propias características de la observación sistemática en el aula, las que permiten el uso del cine como herramienta de entrenamiento en la adquisición de las competencias que se pretenden con el desarrollo del programa formativo de la misma.

Así, las características de la observación sistemática son:

a) Utiliza técnicas observacionales, las cuales puede ser categorizadas mediante el uso de hojas de registro. Es pues, un método de investigación cuantitativo, lo que permite el tratamiento estadístico de los datos que se obtengan. En nuestro caso, en un visionado de una película de cine o más concretamente de una secuencia de una película, se pueden anticipar las categorías a observar. Así, en la hoja de registro los estudiantes podrán anotar las mismas y también la intensidad con la que perciben cada una de ellas (En el apéndice 1 se presenta la hoja de registro elaborada por los alumnos de Magisterio, con las cuatro categorías más valoradas).

b) Permite registrar conductas de tipo espacial, de lenguaje verbal y no verbal. Ambos constructos, están presentes de forma clara en el lenguaje cinematográfico.

c) Se aplica a cualquier procedimiento empleado para describir sucesos, interacciones y materiales utilizados dentro del aula. Todos ellos pueden ser reproducidos en un filme.

d) Puede ser equipada (por ejemplo, mediante un video) o preparada (por ejemplo, un cuaderno de notas). En ambos casos, también aplicables con un visionado.

e) Se puede realizar en ambiente natural (estudios de etología, comportamiento) o seleccionado (en un aula). Este último caso es el del cine. 


\section{ANÁLISIS Y DISCUSIÓN}

\subsection{Los códigos del lenguaje cinematográfico}

El lenguaje cinematográfico obedece a determinados códigos o reglas, como ocurre en otros lenguajes, si bien no puede hablarse de código con la misma firmeza que en otros contextos. Siguiendo a Carmona (2000) consideramos cuatro códigos cinematográficos. (1) El icónico, los elementos que conforman la imagen. (2) El sonoro, que se refiere al sonido, la música y el silencio, entre otros. (3) El lingüístico, palabra escrita y enunciada y, (4) el no lingüístico, compuesto de gestos, modos y apariencia de los actores.

Dada la importancia de los citados códigos para el propósito principal de esta investigación, la instrucción visual mediante el cine para desarrollar la observación sistemática; nos detenemos a continuación a explicar los códigos, para comprender como dicha clasificación puede ayudar a los alumnos a centrarse en cada uno de ellos para aislar cada fenómeno del conjunto de los componentes que aparecen a la vez en una escena.

\subsection{El código icónico}

Elementos que conforman la imagen, el código icónico, son fundamentales en el filme cinematográfico, en el que se le presta una especial atención. Sin embargo, los propios cineastas mantienen cierta controversia en torno al predominio de la imagen sobre la palabra y viceversa. La primacía de la imagen sobre la palabra está presente en numerosos cineastas.

\subsection{El código sonoro}

Hace referencia a toda la banda sonora de una película, incluidos todos los efectos sonoros y ambientales; desde la palabra en forma de comentario, o en forma de voces, o en diálogos sincronizados; hasta la música y el propio silencio, que se tiene en cuenta por su valor expresivo. Para Chion (1999) la banda sonora cumple una función de complemento de las imágenes. La esencia de lo audiovisual impone la armonía entre sonido e imagen para construir mensajes perceptibles.

\subsection{El código lingüístico}

El código lingüístico se refiere a toda palabra, escrita o enunciada, que aparece en una película. Por ejemplo, el título, los créditos, y cualquier otro texto es código lingüístico escrito. En la palabra enunciada, encontramos la voz en off del narrador, la letra de la música si la tiene, las reflexiones que oímos, los diálogos, entre otros. 


\subsection{El código no lingüístico}

La expresividad de los códigos lingüístico y no lingüístico es empleada por los actores para comunicar su intimidad y su humanidad si apoyan la palabra con el gesto. Para interpretar, el actor cuenta con tres facetas diferenciadas que son: la acción, la mímica y la expresión facial. En otros términos, los elementos visuales (apariencia, gestos) y sonoros (voz, efectos). Incluye además otros aspectos del actor como son la interpretación, el vestuario y el maquillaje.

\subsection{La comunicación no verbal}

La comunicación no verbal, aquella que se expresa con otros elementos distintos a las palabras está presente en todo filme cinematográfico. Utiliza el código no lingüístico mencionado anteriormente. De acuerdo con Pinazo (2001) la comunicación no verbal tiene tres extensiones: la paralingüística, la kinesia y la proxemia, que se dan en el actor.

\subsection{El aprendizaje cooperativo}

Nuestra experiencia con un la impartición de un seminario para profesores sobre "habilidades sociales" y con el desarrollo de actividades con alumnos que facilitan el aprendizaje cooperativo nos animó a volverlo a aplicar en el aula. En la actividad con alumnos, utilizamos la metodología del aprendizaje cooperativo, a través de la dinámica de grupos, en dos fases, la mesa redonda y el debate dirigido por el profesor. Nuestros alumnos fueron capaces de adquirir competencias generales y específicas a través de esta innovación docente, siendo conscientes de su aprendizaje significativo, lo que pudimos comprobar mediante entrevistas semi estructuradas (Parra \& Peña, 2009).

En el contexto que nos ocupa el aprendizaje cooperativo se entiende como "un concepto diferente del proceso de enseñanza y aprendizaje. Se basa en la interacción entre alumnos diversos, que en grupos de 4 a 6, cooperan en el aprendizaje de distintas cuestiones de índole muy variada. Este aprendizaje cuenta con la ayuda del profesor, que dirige este proceso supervisándolo. Se trata, pues, de un concepto del aprendizaje no competitivo ni individualista como lo es el método tradicional, sino un mecanismo colaborador que pretende desarrollar hábitos de trabajo en equipo, la solidaridad entre compañeros, y que los alumnos intervengan autónomamente en su proceso de aprendizaje" (EducaMadrid, 2010). 
En definitiva, el aprendizaje cooperativo "consiste en trabajar juntos para alcanzar objetivos comunes. En una situación cooperativa, los individuos procuran obtener resultados que sean beneficiosos para ellos mismos y para todos los demás miembros del grupo. El aprendizaje cooperativo es el empleo didáctico de grupos reducidos en los que los alumnos trabajan juntos para maximizar su propio aprendizaje y el de los demás" (Johnson et al., 1999).

\section{CONCLUSIONES}

El proceso de enseñanza-aprendizaje de una materia científica, la observación sistemática, que suele abordarse como una asignatura teórica se puede complementar de forma práctica con un enfoque multidisciplinar. En este caso se emprendió a través de dos disciplinas propias de las Ciencias de la Comunicación:

El Lenguaje Cinematográfico y La Comunicación no verbal. Esta metodología interdisciplinar permite al estudiante obtener una visión más completa de la materia objeto de estudio, lo que contribuye a detectar actitudes, uno de los fines perseguidos con la observación sistemática. Además, el alumno se convierte en el centro del proceso de enseñanza-aprendizaje, como pretende el EEES.

El trabajo aquí descrito supone un avance en la aplicación de la innovación docente, potenciando el trabajo cooperativo, donde el alumno es consciente de su aportación individual y de la del grupo (MEC, 2005; Lara y Rivas 2009; Villar, 2004). No solo se conoce y domina la técnica de observación sistemática, sino que además se adquiere la capacidad de criterio para comentar un producto audiovisual. Se desarrolla la sensibilidad estética y la admiración por el trabajo laborioso, que cuida los detalles.

Las competencias adquiridas permitieron a los alumnos desarrollar el trabajo autónomo y cooperativo, creando por sí mismo instrumentos de observación sistemática, como una guía de observación. Además, se alcanzaron los objetivos generales de la asignatura, entre los que se puede destacar: Actuar como mediador para que cualquier actividad que se lleve a cabo resulte significativa y estimule el potencial de desarrollo de cada individuo en el trabajo cooperativo. Los alumnos son capaces de desarrollar trabajos disciplinares, propios de la observación sistemática, pero también interdisciplinares combinando aquella con la Cienciade la Comunicación. Por tanto, son capaces de analizar el contexto en el que se desarrolla su actividad, lo que les permite dar respuesta a una sociedad en constante evolución. 
Así mismo, mediante la instrucción audiovisual, los alumnos lograron los objetivos específicos de la materia: conocer las diferentes estrategias de investigación, comprender y aplicar la metodología observacional, y usar su terminología específica.

De forma concreta han recogido, analizado, e interpretado datos observacionales y han valorado e interpretado tanto su propio trabajo como otros de investigación observacional. Por lo que se refiere a las competencias adquiridas los alumnos han demostrado, capacidad de análisis y síntesis, capacidad de gestión de la información, comprender la observación sistemática como instrumento básico de reflexión sobre la realidad, contribuyendo a la innovación docente, mediante las prácticas realizadas. En definitiva, son capaces de dominar la técnica de la observación sistemática.

Las destrezas adquiridas por los estudiantes sobre lenguaje audiovisual se pueden aplicar en otros contextos. De hecho, nuestros alumnos participaron en un blog sobre un programa audiovisual sobre educación. La combinación del conocimiento de los códigos cinematográficos, del lenguaje no verbal y de la observación sistemática confiere al individuo la capacidad de criterio para juzgar y opinar por sí mismo en otros ámbitos diferentes al entorno del aula universitaria. De este modo, los conocimientos adquiridos en la universidad son aplicables a su vida social, lo que a largo plazo les capacita para la participación democrática.

Las técnicas de observación sistemática pueden utilizarse para proporcionar retroalimentación acerca de los propios métodos de enseñanza, ya sea durante las clases en el aula o en el período de prácticas del alumno, pero también en situaciones especialmente diseñadas para ello, como las empleadas por la micro enseñanza.

Además, el proceso de actuar como observador puede ayudar a los estudiantes a ser más conscientes de la dinámica de los procesos de enseñanza y de interacción en clase. Por tanto, los alumnos aprenden el proceso que conlleva diseñar un programa de observación, pero también a decidir si un programa existente se adecua a unos propósitos concretos. En definitiva, la observación sistemática puede servir para medir la efectividad de los métodos pedagógicos.

La observación sistemática debe convertirse en una materia propia de la formación del profesorado, puesto que su dominio puede permitir la mejora de nuestra labor como docentes, profundizando en la idea del profesor como investigador, adquiriendo así espíritu crítico y recursos para supervisar el trabajo de nuestros alumnos. 


\section{REFERENCIAS}

AAVV. (2010). Diccionario on-line profes.net. Recuperado el 16 de septiembre de 2010, de http://clave.librosvivos.net/

AAVV. Glosario de términos psicológicos APA. Recuperado el 14 de octubre de 2010, de http://www.apa.org/research/action/glossary.aspx\#o

Almacellas Bernado, M. A. (2004). Educar con el cine. Barcelona: EIUNSA.

Almacellas Bernado, Ma A. \& Piscitello, T. (2000). Educar la inteligencia. Descubrimiento de los valores a través de la literatura y el cine. Córdoba: Galeón.

ANECA. (2005). Libro Blanco, Título de Grado en Economía y Empresa. Madrid: Agencia Nacional de Evaluación de la Calidad y Acreditación.

Carmona, R. (2000). Cómo se comenta un texto fílmico. Madrid: Cátedra.

Comey, J. (2002). The art of film acting. Nueva York: Focal Press.

Croll, P. (1995). La observación sistemática en el aula. Barcelona: editorial la Muralla.

Chion, M. (1999). El sonido. Barcelona: Paidós.

De La Torre, S. (1996). Cine formativo. Barcelona: Octaedro.

De Pablo Pons, J. (1986). Cine y enseñanza. Madrid: Ministerio de Educación y Ciencia.

De Pablo Pons, J. P. (1998). Nuevas Tecnologías, Comunicación Audiovisual y Educación. Barcelona: Cedecs.

Hall, E. T. (1992). La dimensión oculta. México: Siglo XXI Editores.

Lara, S. \& Rivas, S. (2009). Aprendizaje autorregulado y fomento de competencias en dos asignaturas de master a través del empleo de plantillas de evaluación, método del caso, role-playing y video digital. Educación XX1(12): 67-96.

Le Boterf, G. (2001). Ingeniería de las competencias. Barcelona: Gestión 2000.

Ministerio de Educación y Ciencia (MEC). (2005). Espacio Europeo de Educación Superior. Madrid: Consejo de Coordinación Universitaria.

Morales Vallejo, P. (2006). Medición de actitudes en psicología y educación. Madrid: Universidad Pontificia de Comillas. 
Peña Acuña, B. (2010). Video forums. Portal educativo PROFES NET. Recuperado el 19 de julio de 2010, de http:/ / www.religion.profes.net/propuestas2.asp?ciclo $=4205 \&$ categoria $=39210 \&$ nom bre_id=Valores+en+el+cine\&id_categoria $=140 \& c a t=S e g u n d o+C i c l o+E S O$

Peña Acuña, B. (2004).Vertiente educativa del cine. Trabajo presentado al Congreso Edutec. Recuperado el 20 de junio de 2010, de http:/ / www.edutec2004.lmi.ub.es/pdf157.pdf

Peña Acuña, B. (2010). Humanismo y cine: el tratamiento de la dignidad humana en la obra de Steven Spielberg. Alicante: Universidad de Alicante. Tesis inédita.

Pinazo, D. (2010).La Comunicación no verbal. Recuperado el 23 de junio de 2010, de http://www3.uji.es/ pinazo/2001Tema\%205.htm

Parra MeroñO, M. C. \& Peña Acuña, B. (2009). Participatory activities for cooperative learning. Trabajo presentado al Congreso Internacional de Educación, Investigación e Innovación. Recuperado el 23 de junio de 2010, de http:/ / www.iated.org/concrete2/session_detail.php?session_id=295

EDUCAMADRID. (2010). Portal educativo. Recuperado el 23 de junio de 2010, de http://www.educa.madrid.org/portal/c/portal/layout?p_1_id=13380.148

UCAM. (2009). Guía académica del Grado de Magisterio Infantil. Disponible en CD. Murcia.

Villar, L. M. (2004). Programa para la mejora de la docencia universitaria. Madrid: Pearson/Prentice Hall. 


\section{Beatriz Peña-Acuña}

Es doctora por la Universidad de Alicante. Licenciada en Filología, Diplomada en Humanidades y Periodista. Profesora de "Observación sistemática y análisis de contextos" en Magisterio Infantil, "Trabajo fin de Grado" en Turismo y "Comunicación interna" a posgrados. Profesora del Cuerpo de Tutores de la Universidad Católica San Antonio. Ha participado en diversos congresos internacionales de innovación educativa como International Conference of Education and Innovation e International Technology, Education and Development Conference con varias ponencias en inglés y ha publicado artículos concernientes a la educación en revistas de Ciencias Sociales como el Journal of Alternative Perspectives y Vivat Academia. Ha publicado material para docentes de Secundaria para la editorial online de Santillana: profes.net. Ha publicado un manual sobre "Métodos de observación aplicados a las Ciencias Sociales y la Educación" dirigido a alumnos de Magisterio.

\section{Concepción Parra-Meroño}

Es doctora en Administración y Dirección de Empresas. Profesora de Dirección Comercial y Marketing. Profesora del Cuerpo de Tutores de la Universidad Católica San Antonio. Ha participado en diversos congresos internacionales de educación como International Conference of Education and Innovation e International Technology, Education and Development Conference con varias ponencias y ha publicado artículos científicos en revistas de educación como Estudios Sobre Educación y en otras revistas de Ciencias Sociales como Estudios sobre Consumo y Cuadernos de Economía y Dirección de la Empresa. Además es autora de varios capítulos de libro para la docencia basados en metodologías innovadoras, como el método del caso. 